\title{
Bile Salt-Stimulated Lipase and Pancreatic Lipase-Related Protein 2 Are the Dominating Lipases in Neonatal Fat Digestion in Mice and Rats
}

\author{
XIAONAN LI, SUSANNE LINDQUIST, MARK LOWE, LAILA NOPPA, AND OLLE HERNELL
}

Department of Clinical Sciences [X.L., S.L., L.N., O.H.], Pediatrics, Umeå University, SE-901 87 Umeå, Sweden; Nanjing Children's Hospital [X.L.], Nanjing Medical University, Nanjing 210008, P.R. China; Department of Pediatrics [M.L.], Children's Hospital of Pittsburgh at UPMC, Pittsburgh, Pennsylvania 15213; Department of Medical Countermeasures [L.N.], Division of CBRN Defence and Security, Swedish Defence Research Agency, SE-901 82 Umeå, Sweden

\begin{abstract}
During infancy, the basic conditions for digestion of dietary fat differ from later in life. The bile salt-stimulated lipase (BSSL) is an enzyme expressed in the exocrine pancreas and in some species (including human) also in the lactating mammary gland and secreted with the milk. The aim of this study was to compare the ontogeny of four pancreatic lipases [BSSL, pancreatic triglyceride lipase (PL), pancreatic lipase-related protein 2 (PLRP2), and phospholipase A2 (PLA2)] in one species that supplies BSSL with milk (the mouse) and one that does not (the rat). We followed expression of the four pancreatic lipases from postnatal d 1 until after weaning in both species. We found that BSSL and PLRP2, two lipases with broad substrate specificity, dominated. It was not until weaning that significant expression of PL and PLA2 were induced. Thus, BSSL and PLRP2 seem to be responsible for fat digestion as long as milk is the main food. Moreover, the early temporal pattern of BSSL expression differed between species. We speculate that the milkborne BSSL is able to compensate for a slower ontogeny of pancreatic BSSL expression in the mouse. (Pediatr Res 62: 537-541, 2007)
\end{abstract}

$\mathrm{T}^{\mathrm{s}}$ The exocrine pancreas synthesizes and secretes several lipolytic enzymes that operate in concerted action with gastric lipase to achieve complete digestion and absorption of dietary fat (1). In adults, pancreatic triglyceride lipase (PL) is the major enzyme responsible for intestinal digestion of dietary triglycerides. In fact, in the presence of colipase and bile salts, PL has been estimated to account for $56 \%$ of the intraluminal hydrolysis of dietary triglycerides in adults (2). PL is mainly active on tri- and diglycerides, whereas phospholipase A2 (PLA2) is the major enzyme involved in hydrolysis of dietary phospholipids in adults (3).

During infancy, the digestive system continues to develop and the basic conditions for fat digestion are different from those later in life. The intraluminal level of bile salts is low, particularly in preterm infants (4), and expression of PL in neonates is also low, or undetectable, until the suckling-

Received March 14, 2007; accepted June 19, 2007

Correspondence: Susanne Lindquist, Ph.D., Department of Clinical Sciences, Pediatrics, Umeå University, SE-901 87 Umeå, Sweden; e-mail: susanne.lindquist@pediatri. umu.se

This work was supported by grants from the Swedish Research Council, Medicine, Her Royal Highness the Crown Princess Lovisa's Society for Pediatric Research/Axel Thielman's Memorial Fund, Västerbotten County Council (ALF), Oskarfonden and Biovitrum AB. weanling transition (5-8). However, two pancreatic lipaserelated proteins (PLRP1 and PLRP2) are expressed before birth, and expression persists during the suckling period and into adulthood (5-7). The amino acid sequence of PLRP1 and PLRP2 shows a high degree of homology (65\%-70\%) with PL, but their functional properties vary substantially. PLRP2 presents a broader substrate specificity compared with PL and hydrolyses not only triglycerides but also galactolipids, phospholipids, and retinyl palmitate (9-12). However, in contrast to PL, the activity of PLRP2 on triglycerides is inhibited by physiologic bile salt concentrations, even in the presence of colipase $(10,11)$. In accord with the high expression during infancy, PLRP2 has been shown to perform a crucial role in the digestion of dietary fat in suckling mice. PLRP2-deficient pups developed steatorrhea, and their stools contained large quantities of incompletely digested dietary fat $(6,13)$. PLRP1 displays no recognized lipolytic activity and has no known physiologic function. Two amino acid substitutions, V179A and A181P, compared with PL render the protein inactive (14).

Bile salt-stimulated lipase (BSSL) is another important enzyme involved in digestion and absorption of dietary fat. Similar to PLRP2, BSSL has the capacity to hydrolyze a variety of substrates, e.g. tri-, di-, and monoglycerides; cholesteryl esters; fat-soluble vitamin esters; phospholipids; galactolipids; and ceramides (15-17). BSSL is expressed in the exocrine pancreas in all species, and in some species (including the human and the mouse, but not the rat), it is also expressed in lactating mammary glands and secreted with the milk (18-20). Milk-derived BSSL contributes significantly to the efficient utilization of milk fat in newborn infants (1). Depletion of BSSL in genetically modified mice caused fat malabsorption and subsequent fat-derived epithelial disruption in the distal ileum (21). Interestingly, when foster dams expressing active BSSL in their milk nursed the BSSLdeficient pups, fat absorption was restored to normal and no intestinal lesions were found.

Abbreviations: BSSL, bile salt-stimulated lipase (EC 3.1.1.3); PL, pancreatic triglyceride lipase (EC 3.1.1.3); PLA2, phospholipase A2 (EC 3.1.1.4); PLRP2, pancreatic lipase-related protein 2 (EC 3.1.1.3) 
Hence, milk supplied-BSSL contributes to efficient digestion of milk fat in species expressing BSSL in the mammary glands during lactation. Obviously, this cannot be the case in species not secreting BSSL with the milk. We hypothesized that expression of pancreatic lipases during the suckling period differs between species that have BSSL supplied with the milk and species that do not. Thus, we compared the ontogeny of the lipases secreted by the exocrine pancreas in the mouse, which has BSSL in milk, and the rat, which does not. We report on a significant divergent temporal expression that supports a view that BSSL, together with PLRP2, are the dominating lipases involved in fat digestion as long as milk is the main food.

\section{MATERIALS AND METHODS}

Animal procedures. Balb/c/Bkl mice and Sprague-Dawley rats were purchased from Scanbur AB (Sollentuna, Sweden) and bred in-house. The animals were kept in rooms with a 12:12-h light-dark cycle and with unrestricted access to R34 standard chow diet (Lactamin, Stockholm, Sweden) and water. All animal procedures were approved by the regional Committee on Ethics in Experimental Science using Animals, Umeå, Sweden.

At different time points, i.e. at postnatal d 1, 2, 3, 4, 5, 7, 10, 12, 16, 20, $24,28,32$, and at 6 mo of age (for RNA) and at postnatal d 1, 7, 14, 21, and 28 (for protein), at least three mice and three rats were killed by cervical dislocation and the pancreas was immediately carefully removed for RNA and protein isolation. Before sacrifice, animals were observed when having a meal (milk or chow) and subsequently immediately moved to a cage without food (only water) for a 2-h fasting period.

Quantification of $\boldsymbol{m R N A}$ expression levels. TRIzol Reagent (Invitrogen, Carlsbad, CA) was used to isolate total RNA from whole pancreas obtained from newborn mice and rats at postnatal d $1-5$ or pieces of pancreatic tissue (100-200 mg) from older animals. The RNA was stored at $-70^{\circ} \mathrm{C}$ until use. First strand cDNA was synthesized from total RNA using TaqMan reverse transcription reagents (Applied Biosystems, Foster City, CA) and random hexamers, following the manufacturer's recommendations. Quantitative realtime polymerase chain reaction analysis was performed using the ABI PRISM 7000 Sequence Detection System and TaqMan universal PCR master mix (Applied Biosystems) according to the manufacturer's recommendations. All primers and probes (Table 1) were designed using Primer Express 1.0 software (Applied Biosystems), according to guidelines. Samples were run in triplicate. Expression of $18 \mathrm{~S}$ rRNA was determined in all samples using predeveloped TaqMan assay reagents (Applied Biosystem) and used as an endogenous control to normalize expression levels. mRNA values obtained for BSSL, PLRP2, PTL, and PLA2 were presented as a ratio of target gene/18S rRNA.

Protein extraction and Western blots. Pieces of pancreatic tissue (10-100 $\mathrm{mg}$ ) obtained from mice and rats as described above on postnatal d 1, 7, 14, 21 , and 28 were homogenized in protein lysis buffer [50 mM Tris, $0.5 \%$ Nonidet P-40, $0.5 \%$ Na-deoxycholate, $0.1 \%$ sodium dodecyl sulfate, $150 \mathrm{mM}$ $\mathrm{NaCl}, 1 \mathrm{mM}$ ethyldiamine tetraacetic acid, $\mathrm{pH}$ 7.5] containing 1 Mini Complete tablet per $10 \mathrm{~mL}$ (Roche Diagnostics, Mannheim, Germany)]. The homogenate was centrifuged at $14,000 \mathrm{rpm}$ for $10 \mathrm{~min}$, and the supernatant was collected. All steps were performed at $4^{\circ} \mathrm{C}$ to minimize the risk of proteolysis. The protein concentration in each sample was determined using the BCA Protein Assay (Pierce, Rockford, IL), following the manufacturer's recommendations. Thirty micrograms of total protein (for PLA2 $50 \mu \mathrm{g}$ ) was separated on $7.5 \%$ (BSSL), 10\% (PLRP2 and PL), or 15\% (PLA2) sodium dodecyl sulfate-polyacrylamide gel (SDS-PAGE) electrophoresis according to Laemmli (22). Protein was transferred to polyvinylidene difluoride (PVDF) membranes (Bio-Rad, Hercules, CA) according to usual techniques and Western blotting was carried out using the ECL Advance Western Blotting Detection kit, following the manufacturer's recommendations (GE Healthcare, Buckinghamshire, UK). Polyclonal anti-human BSSL antibodies and anti-PLRP2 peptide antibodies were raised in rabbits and purified as previously described $(6,23)$. Goat anti-human PL and rabbit anti-porcine PLA2 were purchased from Nordic Biosite AB (TÄBY, Sweden) and Upstate Biotechnology (Lake Placid, NY), respectively. Peroxidase-conjugated donkey anti-rabbit IgG or rabbit anti-goat IgG (DAKO, Glostrup, Denmark) were used as secondary antibodies. The positive controls on the Western blot were enzymes from the following sources: BSSL isolated from human milk (24), recombinant rat PLRP2 (9), PL isolated from human pancreas (purchased from Sigma Chemical Co.-Aldrich, St. Louis, MO), and PLA2 from porcine pancreas (purchased from Fluka, Buchs SG, Schweiz).

Statistics. All data are presented as means \pm SEM, and all statistical analyses were carried out using Statistical Package for Social Sciences (SPSS). Comparisons of several means were done by one-way analysis of variance with post hoc multiple comparison (least significant difference). A $p$ value $<0.05$ was considered significant.

\section{RESULTS}

Expression of the four pancreatic lipases (BSSL, PL, PLRP2, and PLA2) was followed in mice and rats by real-time quantitative PCR and Western blots from postnatal d 1 into adulthood. Although mRNA encoding BSSL was detected from d 1 in both species, a clear difference in the pattern of expression was found during the first weeks postpartum (Fig. $1 A)$. In mice, the level of BSSL mRNA increased significantly from $\mathrm{d} 4$ to peak around $\mathrm{d} 7$ (d 1-3 versus $\mathrm{d} 5-12, p<0.05$ ); thereafter, it declined successively reaching adult levels during the suckling-weaning transition around d 16-20. In contrast, rat pancreatic BSSL mRNA was expressed at relatively constant level from d 1 until adulthood (no significant difference between $\mathrm{d} 1-180$ ).

The patterns of early BSSL expression in the pancreas of mice and rats were confirmed by Western blots (Fig. 1B). For both species, the BSSL protein was clearly detectable by $\mathrm{d} 1$. In accord with the recorded mRNA levels, the amount of BSSL protein in mouse pancreas increased during the first week, peaked between d 7 and 14, and subsequently declined at the time of weaning. In rats, the amount of BSSL protein present in pancreas at d 1 appeared to be higher compared with mice and did not vary over time from birth to after

Table 1. Primers and probes used for quantitative PCR

\begin{tabular}{|c|c|c|}
\hline Gene & Mouse & Rat \\
\hline \multirow{3}{*}{$B S S L$} & Forward: 5'-TGCGTGTCTGAAGATCACAGA-3' & Forward: 5'-ATCCCCGAGCCTTGACACT-3' \\
\hline & Reverse: 5'-GGATCATCGGGAATGAAGTC-3' & Reverse: 5'-GACAGGGATGAAGGCCAGGT-3' \\
\hline & Probe: 5'-AGTGCACAACAGGGTACTCCTGCTTTTT-3' & Probe: 5'-AAAAGCCAGGAGTACCCCATTGTGCAC-3' \\
\hline \multirow{2}{*}{$P L R P 2$} & Reverse: 5'-TTTTGGGACACCCTTGTTCTG-3' & Reverse: 5'-CCCCTCAAATTGATCAGCATAGT-3' \\
\hline & Probe: 5'-AAGTTCCAGCACAATGACTGCTTCCCTTC-3' & Probe: 5'-CCATTTTGGGACACCCTTCTTCTGGACA-3' \\
\hline$P L$ & Forward: 5' -ATCCGCCACGATGCTAATG-3' & Forward: 5'-GGCAGGAAAGAGGACATTCG-3' \\
\hline \multirow{3}{*}{ PLA2 } & Forward: 5'-GCACCCCAGTGGACGACTT-3' & Forward: 5'-GCACCCCAGTGGACGACTT-3' \\
\hline & Reverse: 5'-TCCAGCTTCTTGGCCTGACT-3' & Reverse: 5'-TTTCCAGCTTCTTGGCCTGAT-3' \\
\hline & Probe: 5'-ACAGGTGCTGCCAGACTCATGACCA-3' & Probe: 5'-ACAGGTGCTGCCAGACTCATGACCA-3' \\
\hline
\end{tabular}



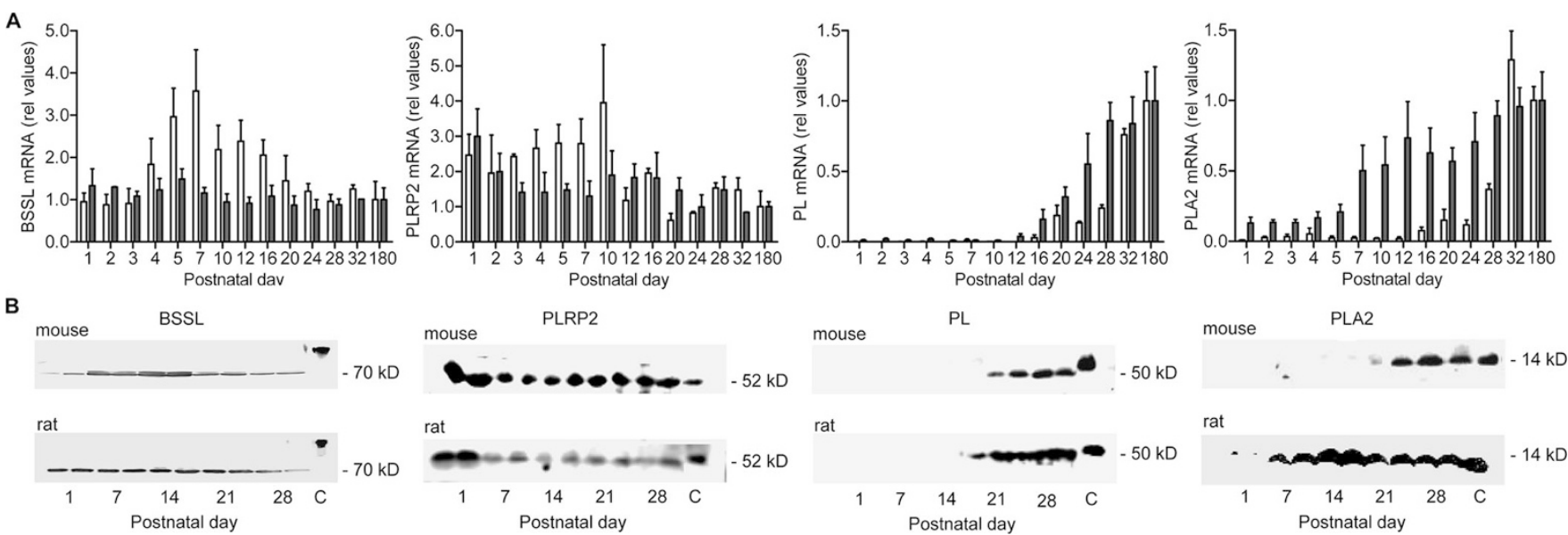

Figure 1. mRNA and protein expression of the pancreatic lipases BSSL, PLRP2, PL, and PLA2 in mouse and rat at different postnatal ages. (A) Relative amount of pancreatic lipase mRNA levels. Bars represent the average values \pm SEM from the analysis of at least three animals at each time point. For each sample, the results were normalized to endogenous $18 \mathrm{~S}$ ribosomal RNA and the adult value for each lipase was set to 1.0. Mouse ( $\square$ ), rat $(\square)$. (B) Immunoblot. Extracts of total pancreatic protein ( $30 \mu \mathrm{g} / \mathrm{lane}$; for PLA2, $50 \mu \mathrm{g} / \mathrm{lane}$ ) was separated by SDS-PAGE, transferred to PVDF membranes and blotted with antibodies directed against pancreatic lipases as noted. C, positive control.

weaning. Note that the positive control used for BSSL in Figure $1 B$ was isolated from human milk. The molecular weight of BSSL differ between species and the human enzyme is significantly larger compared with BSSL found in the mouse and the rat (approximately $110 \mathrm{kD}$ compared with $70 \mathrm{kD}$ ).

Similar to BSSL, pronounced expression of PLRP2 was detected in the pancreas of both mice and rats already at $\mathrm{d} 1$ (Fig. 1A). In mice, mRNA levels were found at a constant level during the first $1.5 \mathrm{wk}$ of life, but decreased at the time the animals start to taste chow diet, i.e., at approximately $\mathrm{d} 12$. In rats, the initial high levels of PLRP2 mRNA decreased at approximately d 2 or 3 postpartum to a level that remained approximately constant until adulthood. In both mice and rats, the amount of PLRP2 protein present in pancreas was found to be highest on postpartum d 1 , after which it rapidly decreased to a lower, approximately constant level (Fig. 1B).

In contrast to BSSL and PLRP2, no significant expression of PL was detected during the first 2 wk of life in either mice or rats (Fig. 1A). Significant levels of PL mRNA were first detected at d 16-20 and reached adult levels approximately 1 wk later at d 28-32. In agreement with mRNA data, the PL protein was not detected in pancreatic homogenates obtained before d 21 (Fig. 1B).

Similar to PL, no significant expression of PLA2 was detected during the first period of life in either mice or rats. However, the time at onset of PLA2 expression in pancreas differed between species. In mice, PLA2 mRNA started to increase by the time of weaning ( $16-20)$ and reached adult levels between d 28 and 32 (Fig. 1A). Mice PLA2 protein was not detected in pancreatic homogenates before d 28 (Fig. 1B). In rats, expression of pancreatic PLA2 started to increase approximately 2 wk earlier (d 5-7; Fig. 1), and the protein was detected already at d 7 (Fig. 1B).

\section{DISCUSSION}

In species expressing BSSL in the lactating mammary glands, the milk-borne enzyme is a prerequisite for efficient digestion of milk fat during the suckling period $(21,25)$. Therefore, we hypothesized that expression of endogenous pancreatic lipases during early infancy differ between species having BSSL supplied with the milk and species that do not and tested this hypothesis by comparing ontogeny of pancreatic lipases in one species that has BSSL supplied with the milk (the mouse) and one that does not (the rat).

BSSL mRNA and protein was detected in the pancreas of mice and rats from postnatal $\mathrm{d} 1$. However, the temporal pattern of expression differed between the two species. In mice, expression increased significantly after the first $4 \mathrm{~d}$ of life. Both mRNA and protein levels reached a peak value at approximately $\mathrm{d} 7$; thereafter, it declined to adult level around weaning. In contrast, BSSL in rats was constitutively expressed from birth into adulthood. It is tempting to speculate that the BSSL expression in the mouse (but not in rat) is insufficient at birth and during the first postnatal days, but that the milk-borne BSSL is able to compensate for the slower ontogeny of pancreatic BSSL expression in suckling mouse pups. This hypothesis is supported by a previous study suggesting a developmental window during the first week of life when the supply of milk BSSL is most critical (21). BSSL heterozygous pups nursed by BSSL-deficient dams developed disruption of the epithelium due to massive accumulation of undigested lipids in the distal part of the small intestine. This problem was most prominent before postnatal d 5, which, according to our present data, coincides with ontogeny of pancreatic BSSL.

The gradual induction of BSSL mRNA in mouse pancreas during the first week of life may be a response to the onset of feeding. A similar increase of mRNA expression immediately after birth was previously reported for PLRP1, PLRP2, and colipase in rat pancreas (5). Our present study confirms that PLRP2 mRNA and protein levels are higher at d 1-7 compared with later in life, although we did not observe any additional induction after birth. Exactly what triggers the response to onset of feeding is not known, but expression of 
pancreatic lipases, including BSSL, PLRP2, and PL, are all regulated by the amount and/or type of dietary fat (26-29). However, the response to dietary fat differs between genes encoding the different pancreatic lipases. In suckling rats, PL and PLRP2 transcription was shown to be anticoordinately regulated by amount of dietary polyunsaturated fatty acids (PUFAs). High levels of PUFAs significantly down-regulated the PLRP2 mRNA expression during the preweaning phase (28). The BSSL gene expression is not regulated by dietary triglycerides, but by high cholesterol levels in the diet (29).

Leptin, a hormone involved in regulation of food intake and energy metabolism, was recently shown to decrease PL and PLRP1 transcription and to increase PLRP2 transcription in the exocrine pancreatic cell line AR42J (30). Because leptin is present in human milk (31), serum concentration is higher in breast-fed than in formula-fed infants (32) and lower in colostrum compared with mature milk, in which it decreases with time of lactation (33), it is tempting to speculate that a high concentration of milk leptin during the initial period of suckling (except the first days) increases serum leptin levels and induces PLRP2 and suppresses PL transcription. However, further studies are needed to explore the effect of leptin on pancreatic lipases in vivo and whether leptin affects BSSL expression.

It is an intriguing question why BSSL and PLRP2, two nonspecific lipases with broad substrate specificities, seem to be the key players in fat digestion as long as milk is the main food. During infancy, the intraluminal level of bile salt is low compared with later in life (4), and we know from earlier studies that milk fat globules are a poor substrate for PL even in the presence of colipase and bile salts $(34,35)$. Conversely, the triglyceride activity of human PLRP2 is inhibited by bile salts and addition of colipase does not completely restore the activity (10). Due to the comparatively low bile salt concentration, this may be of less relevance in the newborn. It is tempting to speculate that the concerted action of gastric lipase, PLRP2, and BSSL are ideally suited to efficiently digest human milk fat globules under the intraluminal conditions of infancy. As the digestive system matures and expression of PL and PLA2 increases, the role of BSSL and PLRP2 may change. After weaning, BSSL and PLRP2 may function as complementary lipases to increase the efficiency of dietary fat hydrolysis and absorption. This hypothesis is supported by previous studies on genetically modified mice, in which BSSL and/or PLRP2 were suggested to compensate for the lack of PL and PLA2 activity in catalyzing triglyceride and phospholipid digestion, respectively $(36,37)$. BSSL and PLRP2 could also have more specific functions after weaning. For example, the main physiologic function of PLRP2 has been suggested to be to hydrolyze galactolipids $(10,11)$, a substrate also hydrolyzed by BSSL. BSSL has been shown to play a primary role in absorption of cholesterol esters $(38,39)$. Compared with infant formula, human milk is rich in cholesterol and the endogenous cholesterol synthesis rate is lower in breast-fed than in formula-fed infants (40). When infants are fed an experimental formula supplemented with cholesterol to meet the concentration of human milk, the synthesis rate is reduced but not to the level of breast-fed infants (41). This suggests that factors other than the cholesterol concentration in milk affect cholesterol metabolism in breast-fed infants. Duration of breast-feeding is negatively associated to serum cholesterol levels later in life, and a programming effect on later cholesterol metabolism has been proposed (42). It is possible that milk-borne BSSL could affect the cholesterol metabolism and influence the mechanism underlying the programming effect (39).

To our knowledge, this is the first report showing the temporal, developmental pattern of BSSL expression in the pancreas of mice and rats. We also confirmed and extended previous studies in rodents and humans showing a divergent temporal expression of PL and PLRP2 during the suckling period (5-7). Our data clearly support and add to previous findings that it is BSSL together with PLRP2 that are the dominating pancreatic lipases during the first weeks of life. It is not until weaning (at approximately d 16-20) that significant expression of classic PL and PLA2 are induced. Further studies will focus on the concerted action of lipolytic enzymes under conditions resembling those in vivo of preterm infants. The developmental pattern of expression described in this study may have important consequences for nutritional therapy of preterm infants and newborns.

Acknowledgments. The authors thank Eva-Lotta Vestman and Yvonne Andersson for excellent technical assistance. We are grateful to Assistant Professor Lars Bläckberg for his interest in the work and for fruitful discussions.

\section{REFERENCES}

1. Bernback S, Blackberg L, Hernell O 1990 The complete digestion of human milk triacylglycerol in vitro requires gastric lipase, pancreatic colipase-dependent lipase, and bile salt-stimulated lipase. J Clin Invest 85:1221-1226

2. Carriere F, Barrowman JA, Verger R, Laugier R 1993 Secretion and contribution to lipolysis of gastric and pancreatic lipases during a test meal in humans. Gastroenterology 105:876-888

3. Carey MC, Hernell O 1992 Digestion and absorption of fat. Semin Gastrointest Dis 3:189-208

4. Norman A, Strandvik B, Ojamae O 1972 Bile acids and pancreatic enzymes during absorption in the newborn. Acta Paediatr Scand 61:571-576

5. Payne RM, Sims HF, Jennens ML, Lowe ME 1994 Rat pancreatic lipase and two related proteins: enzymatic properties and mRNA expression during development. Am J Physiol 266:G914-G921

6. Lowe ME, Kaplan MH, Jackson-Grusby L, D'Agostino, D Grusby MJ 1998 Decreased neonatal dietary fat absorption and $\mathrm{T}$ cell cytotoxicity in pancreatic lipase-related protein 2-deficient mice. J Biol Chem 273:31215-31221

7. Yang Y, Sanchez D, Figarella C, Lowe ME 2000 Discoordinate expression of pancreatic lipase and two related proteins in the human fetal pancreas. Pediatr Res 47:184-188

8. Fredrikzon B, Olivecrona T 1978 Decrease of lipase and esterase activities in intestinal contents of newborn infants during test meals. Pediatr Res 12:631-634

9. Roussel A, Yang Y, Ferrato F, Verger R, Cambillau C, Lowe M 1998 Structure and activity of rat pancreatic lipase-related protein 2. J Biol Chem 273:32121-32128

10. De Caro J, Sias B, Grandval P, Ferrato F, Halimi H, Carriere F, De Caro A 2004 Characterization of pancreatic lipase-related protein 2 isolated from human pancreatic juice. Biochim Biophys Acta 1701:89-99

11. Sias B, Ferrato F, Grandval P, Lafont D, Boullanger P, De Caro A, Leboeuf B, Verger R, Carriere F 2004 Human pancreatic lipase-related protein 2 is a galactolipase. Biochemistry 43:10138-10148

12. Reboul E, Berton A, Moussa M, Kreuzer C, Crenon I, Borel P 2006 Pancreatic lipase and pancreatic lipase-related protein 2 , but not pancreatic lipase-related protein 1, hydrolyze retinyl palmitate in physiological conditions. Biochim Biophys Acta 1761:4-10

13. D'Agostino D, Lowe ME 2004 Pancreatic lipase-related protein 2 is the major colipase-dependent pancreatic lipase in suckling mice. J Nutr 134:132-134

14. Roussel A, de Caro J, Bezzine S, Gastinel L, de Caro A, Carriere F, Leydier S, Verger R, Cambillau C 1998 Reactivation of the totally inactive pancreatic lipase RP1 by structure-predicted point mutations. Proteins 32:523-531

15. Blackberg L, Hernell O 1983 Further characterization of the bile salt-stimulated lipase in human milk. FEBS Lett 157:337-341

16. Nyberg L, Farooqi A, Blackberg L, Duan RD, Nilsson A, Hernell O 1998 Digestion of ceramide by human milk bile salt-stimulated lipase. J Pediatr Gastroenterol Nutr 27:560-567

17. Hui DY, Howles PN 2002 Carboxyl ester lipase: structure-function relationship and physiological role in lipoprotein metabolism and atherosclerosis. J Lipid Res 43:2017-2030 
18. Hernell O, Olivecrona T 1974 Human milk lipases. II. Bile salt-stimulated lipase. Biochim Biophys Acta 369:234-244

19. Lidmer AS, Kannius M, Lundberg L, Bjursell G, Nilsson J 1995 Molecular cloning and characterization of the mouse carboxyl ester lipase gene and evidence for expression in the lactating mammary gland. Genomics 29:115-122

20. Poorkhalkali N, Lidmer AS, Lundberg LG, Dalrymple MA, Gibson Y, Taylor L, Temperley S, Stromqvist M, Helander HF 1998 Bile salt-stimulated lipase (BSSL) distribution in rat, mouse and transgenic mouse expressing human BSSL. Histochem Cell Biol 110:367-376

21. Howles PN, Stemmerman GN, Fenoglio-Preiser CM, Hui DY 1999 Carboxyl ester lipase activity in milk prevents fat-derived intestinal injury in neonatal mice. Am J Physiol 277:G653-G661

22. Laemmli UK 1970 Cleavage of structural proteins during the assembly of the head of bacteriophage T4. Nature 227:680-685

23. Hansson L, Blackberg L, Edlund M, Lundberg L, Stromqvist M, Hernell O 1993 Recombinant human milk bile salt-stimulated lipase. Catalytic activity is retained in the absence of glycosylation and the unique proline-rich repeats. J Biol Chem 268:26692-26698

24. Blackberg L, Hernell O 1981 The bile-salt-stimulated lipase in human milk. Purification and characterization. Eur J Biochem 116:221-225

25. Williamson S, Finucane E, Ellis H, Gamsu HR 1978 Effect of heat treatment of human milk on absorption of nitrogen, fat, sodium, calcium, and phosphorus by preterm infants. Arch Dis Child 53:555-563

26. Ricketts J, Brannon PM 1994 Amount and type of dietary fat regulate pancreatic lipase gene expression in rats. J Nutr 124:1166-1171

27. Birk RZ, Brannon PM 2004 Regulation of pancreatic lipase by dietary medium chain triglycerides in the weanling rat. Pediatr Res 55:921-926

28. Birk RZ, Regan KS, Boyle-Roden E, Brannon PM 2004 Pancreatic lipase and its related protein 2 are regulated by dietary polyunsaturated fat during the postnatal development of rats. Pediatr Res 56:256-262

29. Brodt-Eppley J, Hui DY 1994 Dietary regulation of cholesterol esterase mRNA level in rat pancreas. J Lipid Res 35:27-35

30. Elinson N, Amichay D, Birk RZ 2006 Leptin directly regulates exocrine pancreas lipase and two related proteins in the rat. Br J Nutr 96:691-696
31. Houseknecht KL, McGuire MK, Portocarrero CP, McGuire MA, Beerman K 1997 Leptin is present in human milk and is related to maternal plasma leptin concentration and adiposity. Biochem Biophys Res Commun 240:742-747

32. Savino F, Fissore MF, Grassino EC, Nanni GE, Oggero R, Silvestro L 2005 Ghrelin, leptin and IGF-I levels in breast-fed and formula-fed infants in the first years of life. Acta Paediatr 94:531-537

33. Savino F, Liguori SA, Lupica MM, Fissore MF, Oggero R 2006 Leptin levels in breast-fed infants. Clin Endocrinol (Oxf) 64:597-598

34. Blackberg L, Hernell O, Olivecrona T 1981 Hydrolysis of human milk fat globules by pancreatic lipase: role of colipase, phospholipase A2, and bile salts. J Clin Invest 67:1748-1752

35. Bernback S, Blackberg L, Hernell O 1989 Fatty acids generated by gastric lipase promote human milk triacylglycerol digestion by pancreatic colipase-dependent lipase. Biochim Biophys Acta 1001:286-293

36. Huggins KW, Camarota LM, Howles PN, Hui DY 2003 Pancreatic triglyceride lipase deficiency minimally affects dietary fat absorption but dramatically decreases dietary cholesterol absorption in mice. J Biol Chem 278:42899-42905

37. Richmond BL, Boileau AC, Zheng S, Huggins KW, Granholm NA, Tso P, Hui DY 2001 Compensatory phospholipid digestion is required for cholesterol absorption in pancreatic phospholipase A(2)-deficient mice. Gastroenterology 120:1193-1202

38. Howles PN, Carter CP, Hui DY 1996 Dietary free and esterified cholestero absorption in cholesterol esterase (bile salt-stimulated lipase) gene-targeted mice. J Biol Chem 271:7196-7202

39. Falt H, Hernell O, Blackberg L 2002 Does bile salt-stimulated lipase affect cholesterol uptake when bound to rat intestinal mucosa in vitro? Pediatr Res 52.509-515

40. Wong WW, Hachey DL, Insull W, Opekun AR, Klein PD 1993 Effect of dietary cholesterol on cholesterol synthesis in breast-fed and formula-fed infants. J Lipid Res 34:1403-1411

41. Bayley TM, Alasmi M, Thorkelson T, Krug-Wispe S, Jones PJ, Bulani JL, Tsang RC 1998 Influence of formula versus breast milk on cholesterol synthesis rates in four-month-old infants. Pediatr Res 44:60-67

42. Singhal A, Cole TJ, Fewtrell M, Lucas A 2004 Breastmilk feeding and lipoprotein profile in adolescents born preterm: follow-up of a prospective randomised study. Lancet 363:1571-1578 